\title{
Tensor Integrals
}

\author{
By William Fabian \\ (Received 13th May, 1955.)
}

\section{Introduction.}

A form of integration of tensors will be introduced here, which will preserve the character of a tensor when so integrated.

Let $T_{v_{1} \ldots . v_{q}}^{u_{1} \ldots u_{p}}$ be the components of a given tensor, defined in the co-ordinate system $x^{r}$ at all points of a curve $l$, in a space $V_{n}$ of affine connection $^{1}$ with connection coefficients $L_{j k}^{i}$. We consider the $n^{p+q}$ differential equations

$$
T_{v_{1} \cdots v_{q}}^{u_{1} \cdots u_{p}}=\frac{\delta}{\delta t}\left(\boldsymbol{X}_{v_{1} \cdots v_{q}}^{u_{1} \cdots u_{p}}\right)
$$

where the quantities on the right are the intrinsic derivatives ${ }^{2}$ of the components $X_{v_{1} \ldots v_{q}}^{u_{1} \ldots u_{p}}$ of a tensor with respect to a parameter $t$ which defines $l$.

Definition. If these differential equations have a solution

$$
X_{v_{1} \ldots v_{q}}^{u_{1} \ldots u_{p}}=Z_{v_{1} \ldots v_{q}}^{u_{1} \ldots u_{p}}
$$

(the indices taking the values 1 to $n$ ), then $Z_{v_{1} \cdots v_{q}}^{u_{1} \cdots u_{p}}$ will be called a tensor integral of $T_{v_{1} \ldots v_{q}}^{u_{1} \ldots u_{p}}$ along $l$ with respect to $t$, and will be written $\int T_{v_{1} \cdots v_{q}}^{u_{1} \cdots u_{p}} \delta t$.

2. Properties of tensor integrals.

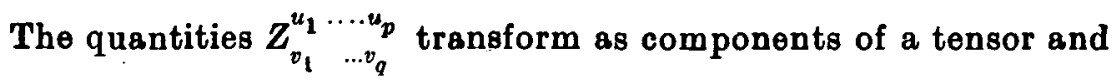

I Eisenhart, Non-Riemannian Geometry (1927), chapter I.

2 These intrinsic derivatives must not be confused with ordinary differential coefficients. For definitions see Eisenhart, op. cit., chapter. I. All quantities used in this paper are real. 
represent therefore a tensor which has the same transformation coefficients as the tensor $T_{{ }_{1} \ldots v_{q}}^{u_{1} \ldots u_{p}}$.

The tensor integral $\int T_{v_{1} \ldots v_{q}}^{u_{1} \ldots u_{p}} \delta t$, if it exists, represents therefore the components of a tensor of the same type and order as the tensor $T_{v_{l} \ldots v_{q}}^{u_{1} \ldots u_{p}}$.

From theorems on differential equations ${ }^{1}$ we can deduce immediately the following two theorems:-

TheоREM 1. If the quantities $d x^{r} / d t$, the coefficients of the connection of the space and the components $T_{v_{1} \ldots v_{q}}^{u_{1} \ldots u_{p}}$ of a tensor in the co-ordinate system $x^{r}$ are continuous funclions of the parameter $t$ which defines $a$ curve l, then a tensor integral $\int T_{v_{1} \ldots v_{q}}^{u_{1} \ldots u_{p}}$ st exists, representing components of a tensor which are continuous along $l$.

ThEOREM 2. If the co-ordinates $x^{r}$, the coefficients of the connection of the space and the components $T_{v_{1} \ldots v_{q}}^{u_{2} \ldots u_{p}}$ of a tensor in this co-ordinate system are analytic functions of the parameter $t$ which defines a curve $l$, then a tensor integral $\int T_{v_{1} \cdots v_{q}}^{u_{1} \cdots u_{p}} \delta t$ exists as a set of analytic functions of $t$ along $l$.

\section{Types of tensor integrals.}

Definition. Quantities $P_{{ }_{1} \cdots v_{q}}^{u \cdots v_{p}}$ satisfying the equations

$$
\frac{\delta}{\delta \ell}\left(X_{v_{1} \ldots . . v_{q}}^{u_{1} \ldots u_{p}}\right)=0
$$

will be said to be the components of tensors parallel with respect to $l$ such that any one of these tensors may be obtained from any other by parallel displacement along this curve.

Hence, if $X_{v \ldots v_{q}}^{u_{1} \ldots u_{p}}=A_{v_{1} \ldots v_{q}}^{u_{1} \ldots u_{p}}$ be a particular solution of the equations

$$
T_{v_{1} \ldots v_{q}}^{u_{1} \ldots u_{p}}=\frac{\delta}{\delta t}\left(X_{v_{1} \ldots v_{q}}^{u 1} \ldots u_{p}\right)
$$

1 Goursat, Mathematical Analysis, translated by Hedrick, vol. II (1916). 
then, by (1), the complete solution of equations (2) is given by

$$
X_{v_{1} \ldots v_{q}}^{u_{1} \ldots u_{p}}=A_{v_{1} \ldots v_{q}}^{u_{1} \ldots u_{p}}-P_{v_{1} \cdots v_{q}}^{u_{1} \ldots u_{p}} .
$$

(a) Thus we obtain the theorem:-

Theorem 3. Under the conditions of either Theorem 1 or Theorem 2, all tensor integrals of $T^{u_{1} \ldots . . u_{v}}$ with respect to $t$ are given by

$$
\int T_{v_{1} \ldots v_{q}}^{u_{1} \ldots u_{p}} \delta t=A_{v_{1} \ldots v_{q}}^{u_{1} \ldots u_{p}}-P_{v_{1} \ldots v_{q}}^{u_{1} \ldots u_{p}},
$$

where $A_{v_{1} \ldots v_{q}}^{u_{1} \ldots v_{p}}$ form a particular tensor integral of the given tensor with respect to $t$, and $P_{v_{1} \ldots v_{q}}^{u_{1} \ldots u_{p}}$ are the components of tensors obtained by parallel displacement along $l$ of an arbitrary initial tensor.

(b) Under the conditions of Theorem 3, we define the tensor integral of $T_{v_{1} \ldots v_{q}}^{u_{1} \ldots \ldots u_{p}}$ along $l$ between the limits $t_{0}$ and $t_{1}$ by

$$
\int_{t_{0}}^{l_{1}} T_{v 1}^{u_{1} \cdots v_{q} \ldots v_{q}} \delta t=\left(A_{v_{1} \ldots v_{q}}^{u_{1} \ldots u_{p}}\right)_{t_{1}}-\left(P_{v_{1} \ldots v_{q}}^{u_{1} \ldots u_{p}}\right)_{t_{1}},
$$

where $\left(A_{v_{1} \ldots v_{p}}^{u_{1} \ldots u_{q}}\right)_{t_{1}}$ are the components of the tensor $A_{v_{1} \ldots v_{q}}^{u_{1} \ldots u_{p}}$ at a point $t_{1}$ on $l$, and $\left(P_{v_{1} \cdots v_{q}}^{u_{1} \cdots u_{p}}\right)_{t_{1}}$ are the components of a tensor at the same point $t_{1}$ obtained by parallel displacement of the tensor $\left(A_{v_{1} \ldots v_{q}}^{u_{1} \cdots u_{p}}\right)_{t_{0}}$ along $l$ from the point $t_{0}$ to $t_{1}$.

(c) The tensor integral of a tensor of order zero is the ordinary integral of the function representing this tensor.

4. Tensor integration by parts.

From the definition and properties of tensor integrals (stated in sections 1 and 2) it follows at once that tensor integration with respect to a parameter defining a curve and intrinsic differentiation with respect to this parameter are inverse operations. 
Theorem 4. Let the quantities $d x^{y} / d t$ and the coefficients of the connection of the space be continuous along a curve l defined by the parameter $t$.

Let the components $U$ of a tensor in the co-ordinate system $x^{r}$ have continuous intrinsic derivatives $\delta U / \delta t$ along $l$, and let the components $W$ of another tensor in this co-ordinate system be continuous along $l$.

Then

$$
\int_{t_{0}}^{t_{1}} U W \delta t=\left(U \int W \delta t\right)_{t_{1}}-(P)_{t_{1}}-\int_{t_{0}}^{t_{1}}\left[\frac{\delta U}{\delta t} \int W \delta t\right] \delta t,
$$

where $(P)_{t_{1}}$ are the components of a tensor at the point $t_{1}$ obtained by parallel displacement of the tensor $\left(U \int W \delta t\right)_{t_{0}}$ along $l$ from the point $t_{0}$ to $t_{1}$.

Proof. $\quad$ Put $\int W \delta t=M$.

We have

$$
\frac{\delta}{\delta t}(U M)=U \frac{\delta M}{\delta t}+M \frac{\delta U}{\delta t}
$$

Hence

$$
\int_{t_{0}}^{t_{1}} \frac{\delta}{\delta t}(U M) \delta t=\int_{t_{0}}^{t_{1}} U \frac{\delta M}{\delta t} \delta t+\int_{t_{0}}^{t_{1}} M \frac{\delta U}{\delta t} \delta t
$$

that is,

$$
(U M)_{t_{1}}-(P)_{t_{1}}=\int_{t_{0}}^{t_{1}} U \frac{\delta M}{\delta t} \delta t+\int_{t_{0}}^{t_{1}} M \frac{\delta U}{\delta t} \delta t
$$

The conclusion follows.

5. The $m^{\text {th }}$ tensor integral of a tensor.

Theorem 5. Let the quaniities $d x^{r} / d t$ and the coefficients of the connection of the space be continuous along a curve $l$ defined by the parameter $t$.

If the components $W$ of a tensor in the co-ordinate system $x^{r}$ are continuous along $l$, then, for all positive integers $m$, the $m^{\text {th }}$ tensor integral of $W$ with respect to $t$ between $t_{0}$ and $z$ is given by

$$
\frac{1}{(m-1) !} \int_{t_{0}}^{z}(z-t)^{m-1} W \delta t .
$$

Proof. $(z-t)^{m-1}$ is a tensor of order zero. Applying Theorem 4, we obtain 


$$
\begin{aligned}
& \frac{1}{(m-1) !} \int_{t_{0}}^{z}(z-1)^{m-1} W \delta t \\
& =\left(\frac{(z-t)^{m-1}}{(m-1) !} \int_{t_{0}}^{t} W \delta t\right)_{z}-(P)+\int_{t_{0}}^{z}\left[\frac{(z-t)^{m-2}}{(m-2) !} \int_{t_{0}}^{t} W \delta t\right] \delta t \\
& =\frac{1}{(m-2) !} \int_{t_{0}}^{z}\left[(z-t)^{m-2} \int_{t_{0}}^{t} W \delta t\right] \delta t,
\end{aligned}
$$

since the components $(P)_{z}$ belong to a tensor obtained by parallel displacement of a zero tensor and are therefore zero for all values of the indices.

Continuing this process of tensor integration by parts, we obtain the conclusion.

\section{Fractional tensor integrals and intrinsic derivatives. ${ }^{1}$}

Now let $m$ have any real value, but let the conditions of Theorem 5 be otherwise satisfied. Then we define the $m^{\text {th }}$ tensor integral, or the $(-m)^{\text {th }}$ intrinsic derivative, of $W$ with respect to $z$ by ${ }^{2}$

$$
\left(\frac{\delta}{\delta z}\right)^{-m} W=\frac{1}{\Gamma(m+c)}\left(\frac{\delta}{\delta z}\right)^{c} \int_{t_{0}}^{z}(z-t)^{m+c-1} W \delta t,
$$

where $c$ is the least integer greater than or equal to zero such that $m+c \geqslant 1$.

Since tensor integration and intrinsic differentiation do not alter the type and order of a tensor, and $(z-t)^{m+c-1}$ is a tensor of order zero, it follows that, for any real value of $m$, the $m^{\text {th }}$ tensor integral $\left(\frac{\delta}{\delta z}\right)^{-m} W$ is a tensor of the same type and order as the tensor $W$.

\section{Tensor expansions. ${ }^{3}$}

Theorem 6. Let the quantities $d x^{r} / d t$ and the coefficients of the connection of the space be continuous along a curve l defined by the para-

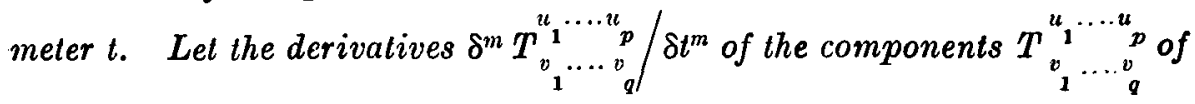

' See my paper in Phil. Mag. (7), XX (1935), 781-789.

2 The gamma function $\mathrm{r}(m+c)$ is not to be confused with the Christoffel symbols.

3 These expansions correspond to the Taylor series for ordinary functions. 
a tensor in the co-ordinate system $x^{r}$ be continuous along $l$ for all positive and zero integers $m$. Then, in the interval of convergence,

$$
\left(T_{v_{1} \cdots v_{q}}^{u_{1} \cdots u_{p}}\right)_{z}=\sum_{m=0}^{\infty} \frac{\left(z-t_{\mathrm{c}}\right)^{m}}{m !}\left(\begin{array}{l}
u_{1} \cdots u_{p} \\
v_{1} \cdots v_{q}
\end{array}\right)_{z},
$$

where $\left({ }^{(m)} U_{v_{1} \cdots v_{q}}^{\prime \prime} \cdots u_{p}\right)_{z}$ are the components of a tensor obtained by parallel displacement of the tensor $\left(\frac{\delta^{m} T_{1}^{u_{1} \ldots u_{p}}}{\delta t^{m}}\right)_{t_{0}}$ along $l$ from $t_{0}$ to $z$.

Proof. . Write $T$ for $T_{v_{1} \ldots v_{q}}^{u_{1} \ldots u_{p}}$, and apply the process of tensor integration by parts of Theorem 4 to the tensor integral $\int_{t_{0}}^{z} 1 T \delta t$. Since 1 is a tensor of order zero (so that its tensor integral is its ordinary integral), we have, by Theorem 4,

$$
\begin{aligned}
\int_{t_{0}}^{z} 1 T \delta t & =(-(z-t) T)_{z}-(P)_{z}+\int_{t_{0}}^{z}(z-t) \frac{\delta T}{\delta t} \delta t \\
= & -(P)_{z}+\int_{t_{0}}^{z}(z-t) \frac{\delta T}{\delta t} \delta t
\end{aligned}
$$

where $(P)_{z}$ is the tensor at the point $z$ obtained by parallel displacement of the tensor $(-(z-t) T)_{t_{0}}$ along $l$ from $t_{0}$ to $z$.

We have also

$$
\left.\begin{array}{rl}
\frac{\delta P}{\delta t} & =0 \\
\frac{\delta}{\delta t}\left({ }^{(0)} U\right) & =0,
\end{array}\right\}
$$

where ${ }^{(0)} U$ stands for ${ }^{(0)} U U_{2}^{u} \ldots u_{p}$

At the point $t=t_{0}$,

$$
P=(-(z-t) T)_{t_{0}}=-\left(z-t_{0}\right)\left({ }^{(0)} U\right)_{t_{0}} .
$$

By (2) and (3), $P=-\left(z-t_{0}\right)\left({ }^{(0)} U\right)$ is a solution of $\delta P / \delta t=0$.

Hence by (1) we have

$\left.\int_{t_{0}}^{z} T \delta t=\left(z-t_{0}\right)\left({ }^{0}\right) U\right)_{2}+\int_{t_{0}}^{z}(z-t) \frac{\delta T}{\delta t} \delta t$ 


$$
\begin{gathered}
=\left(z-t_{0}\right)\left({ }^{(0)} U\right)_{z}+\ldots+\frac{\left(z-t_{0}\right)^{m}}{m !}\left({ }^{(m-1)} U\right)_{\varepsilon}+\ldots \\
\ldots+\int_{t_{0}}^{z} \frac{(z-t)^{m}}{m !} \frac{\delta^{m} T}{\delta t^{m}} \delta t
\end{gathered}
$$

on continuing this tensor integration by parts, where ${ }^{(m-1)} U$ stands for

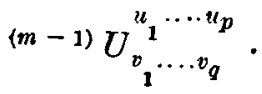

$$
\text { Hence } \begin{aligned}
(T)_{z} & \equiv \frac{\delta}{\delta z} \int_{t_{0}}^{z} T \delta t \\
& =\sum_{n=1}^{m} \frac{\left(z-t_{0}\right)^{n-1}}{(n-1) !}\left({ }^{(n-1)} U\right)_{z}+\frac{\delta}{\delta z} \int_{t_{0}}^{z} \frac{(z-t)^{m}}{m !} \frac{\delta^{m} T}{\delta t^{m}} \delta t
\end{aligned}
$$

This gives the required series, which converges if

when $\boldsymbol{m} \rightarrow \infty$.

$$
\frac{\delta}{\delta z} \int_{t_{0}}^{z} \frac{(z-t)^{m}}{m !} \frac{\delta^{m} T}{\delta t^{m}} \delta t \rightarrow 0
$$

14 Grosvenor Avenue.

Canonbury,

LoNDON, N.5. 